\title{
Reconstruction Algorithm of Signals from Special Samples in Spline Spaces ${ }^{\star}$
}

\author{
Jun Xian ${ }^{1}$ and Degao $\mathrm{Li}^{2}$ \\ ${ }^{1}$ Department of Mathematics, Zhejiang University, \\ Hangzhou, 310027, P.R. China \\ mathxj@163.com \\ ${ }^{2}$ Information Engineering School, Jiaxing University, \\ Jiaxing, 314001, P.R. China
}

\begin{abstract}
In this paper, we introduce integral sampling and study the reconstruction of signals based on non-uniform average samples in spline subspace. By using a new method, we obtain a new reconstruction formula.
\end{abstract}

\section{Introduction}

In digital signal and image processing, digital communication, etc., a continuous signal is usually represented and processed by using its discrete samples. For a bandlimited signal of finite energy, it is completely characterized by its samples, and is described by the famous classical Shannon sampling theorem. However, in many real applications sampling points are not always regular and sampled value may not be values of a signal $f$ precisely at times $x_{k}$ for the inertia of the measurement aparatus. As for the signal spaces, they are not always bandlimited signal of finite energy. The problem arose initially in the design of an interferometer in which the interferogram is obtained using a continuously moving mirror, but may also have bearing in other problems in which the data are smoothed by an integrating sensor, such as a CCD array with slow response time compared to the sample integral. So we need to give the reconstruction of signals from samples of its integral. As special shift-invariant spaces and nonbandlimited spaces, spline subspaces yield many advantages in their generation and numerical treatment so that there are many practical applications for signal or image processing[1-15]. In this paper, we discuss the reconstruction of signal from samples of its integral in spline subspaces.

The outline of this paper is in the following. In Section 2, we introduce the concept of integral sampling and give the reconstruction formula from regular incremental integral samples in spline subspaces. In section 3, numerical results are included. The conclusion is given in Section 4 .

\footnotetext{
* This work is supported in part by the Mathematical Tanyuan Foundation and China Postdoctoral Science Foundation.
} 


\section{Reconstruction of Signal from Regular Incremental Integral Samples in Spline Subspaces}

Suppose $a$ and $b$ are given constants that satisfy $b-a=1$. Let

$$
y_{k}=\int_{-\infty}^{k+b} f(t) d t, y_{k-1}=\int_{-\infty}^{k+a} f(t) d t, z_{k}=y_{k}-y_{k-1} .
$$

We refer to $\left\{z_{k}\right\}$ as the set of regular integral samples. The problem is how to reconstruct the signal $f$ from $\left\{z_{k}\right\}$ (regular incremental integral samples). We now introduce some notations and lemma that will be used in Section 2 . In this paper, the Fourier transform of $f$ is defined by $\hat{f}(\omega)=\int_{\mathbb{R}} f(x) e^{-i x \omega} d x$. The space $V_{N}=\left\{\sum_{k \in Z} c_{k} \varphi_{N}(\cdot-k):\left\{c_{k}\right\} \in \ell^{2}\right\}$ is spline subspace generated by $\varphi_{N}=\chi_{[0,1]} * \cdots * \chi_{[0,1]}(\mathrm{N}$ convolutions), $N \geq 1$. It is well-known that the space $V_{N}$ is a special shift-invariant space.

Lemma 2.1. Let $y(t)=\int_{-\infty}^{t} f(x) d x$ and $f \in V_{N}$, then $y \in V_{N+1}$.

Theorem 2.1 ${ }^{[8]}$. For arbitrary $f \in V_{N}$, we have

$$
f(x)=\sum_{k \in Z} f\left(k+\frac{N+1}{2}\right) S(x-k),
$$

where $\hat{S}(\omega)=\frac{\hat{\varphi}_{N}(\omega)}{\sum \varphi_{N}\left(k+\frac{N+1}{2}\right) e^{-i k \omega}}$ and $V_{N}$ is spline subspace generated by $\varphi_{N}=$ $\chi_{[0,1]} * \cdots * \chi_{[0,1]}(N \geq 1$ convolutions $)$.

By Theorem 2.1 and Lemma 2.1, we have the following result.

Theorem 2.2. Let

$$
y_{k}=\int_{-\infty}^{k+\frac{1}{2}+1} f(t) d t, y_{k-1}=\int_{-\infty}^{k+\frac{1}{2}} f(t) d t, z_{k}=y_{k}-y_{k-1} .
$$

Then for any $f \in V_{N}$, we have reconstruction formula

$$
f(t)=\sum_{k \in Z} z_{k} h(t-k)
$$

where $h$ is defined by

$$
h_{k}=h_{1 k}+h_{k+1}, \hat{h}_{1}(\omega)=i \omega \frac{\hat{\varphi}_{N+1}(\omega)}{\sum \varphi_{N+1}\left(k+\frac{N}{2}+1\right) e^{-i k \omega}},
$$

$h_{k}(\cdot)=h(\cdot-k)$ and $h_{1 k}(\cdot)=h_{1}(\cdot-k)$.

Actually, Theorem 2.2 shows the reconstruction formula from regular incremental integral samples in $V_{N}$. The incremental integral samples can be regarded as special weighted samples. The interpolation function $h$ is implementary. This will be shown in Section 3. 


\section{$3 \quad$ Numerical Examples}

In the section, we will give some numerical tests. So we need a brief and applicable formation of sampling function $h$ in Theorem 2.2 .

In Theorem 2.2, we have relation $h_{k}=h_{1 k}+h_{k+1}$. Taking the Fourier transform of both sides of the above equality, we have the following equality:

$$
\hat{h}(\omega)=\frac{\hat{h}_{1}(\omega)}{1-e^{-i \omega}} .
$$

For precision, we give the following equality

$$
\hat{h}(\omega)=\frac{i \omega}{1-e^{-i \omega}} \frac{\hat{\varphi}_{N+1}(\omega)}{\sum \varphi_{N+1}\left(k+\frac{N}{2}+1\right) e^{-i k \omega}} .
$$

By the inverse Fourier transform, we can find interpolation function $h$. Let $N=3$ in our numerical tests. Then

$$
\hat{h}(\omega)=\frac{i \omega}{1-e^{-i \omega}} \frac{e^{-\frac{4}{2} i \omega}\left(\frac{\sin \frac{\omega}{2}}{\frac{\omega}{2}}\right)^{4}}{\frac{1}{48} e^{2 i \omega}+\frac{23}{48} e^{i \omega}+\frac{1}{24} e^{-i \omega}+\frac{23}{48}}
$$

We will reconstruct the signal $f(x)=\varphi_{3}(x)+2 \varphi_{3}(x-1)+3 \varphi_{3}(x+1) \in V_{3}$ from its integral samples $\left\{z_{k}=\int_{k+\frac{1}{2}}^{k+\frac{3}{2}} f(t) d t\right\}$.

It is obvious that supp $\subseteq \subseteq[-1,4]$. So we only think about finite sampling set, that is, $k=-1,0,1,2,3,4$ in reconstruction formula. The sample values of the function $f$ in integer point $\{f(k)\}_{k=-1,0,1,2,3,4}$ are marked by $*$ in Figure 1. In fact, $\left\{z_{k}=\langle f, u(\cdot-k)\rangle\right\}$ is the result of $\{f(k)\}_{k=-1,0,1,2,3,4}$ perturbed by noise. The noisy signal sampling points $\left\{z_{k}\right\}$ marked by $\square$ and are connected by "- -" in Figure 1.

We will give the reconstruction from the weighted sampling point $\left\{z_{k}\right\}$. In Figure 2, the original signal is represented by continuous line. Reconstruction signal using the above mentioned algorithm is represented by "-..".

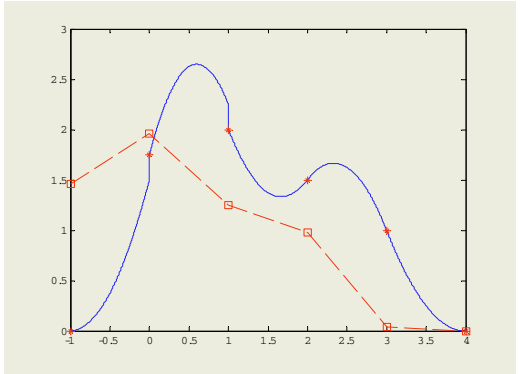

Fig. 1. The noisy signal sampling point $\left\{z_{k}\right\}$ marked by $\square$ and connected by "-". $\{f(k)\}_{k=-1,0,1,2,3,4}$ marked by "*"

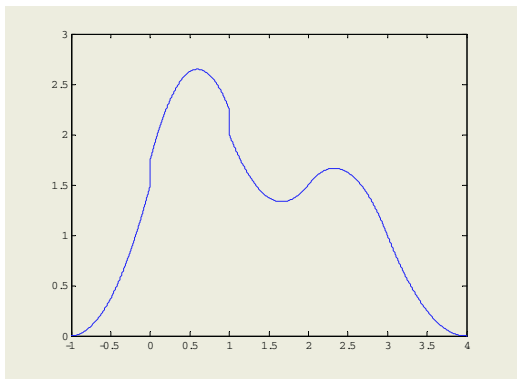

Fig. 2. The original signal is represented by continuous line. The reconstruction signal is represented by "-.." 
To measure the accuracy of the reconstruction, we compute the mean square error(MSE) by the formula $M S E=\sum_{t \in D}\left(f(t)-\sum_{k=-1}^{4} z_{k} \varphi_{3}(t-k)\right)^{2}$, where $D=$ $\{-1,-0.5,0,0.5,1,1.5,2,2.5,3,3.5,4\}$. It is easy to know MSE of the function $f(t)$ is $1.5466 \mathrm{e}-15$.

The graph of the reconstruction signal is almost identical to the graph of the original signal in Figure 2. MSE reflects the observation.

\section{Conclusion}

In this paper, we introduce the new concept of integral sampling where a signal is perfectly reconstructed from the samples of the integral of the signal. The explicit reconstruction formula is obtained in the case of regular sampling in the spline shift-invariant spaces. Finally we successfully give a numerical example to demonstrate out results. Due to the limitation of four-page papers, we omit all proofs.

\section{References}

1. Aldroubi, A., Gröchenig, K.: Beuling-Landau-type theorems for non-uniform sampling in shift invariant spline spaces. J. Fourier. Anal. Appl. 6 (2000) 93-103

2. Aldroubi, A., Gröchenig, K.: Non-uniform sampling and reconstruction in shiftinvariant spaces, SIAM Rev. 43 (2001) 585-620, .

3. Aldroubi, A., Unser, M., Eden, M.: Cardinal spline filters: Stability and convergence to the ideal sinc interpolator. Singal. Processing. 28 (1992) 127-138

4. Chui, C. K.: An introduction to Wavelet, Academic Press, New York,1992

5. Jerri, A. J.: The Gibbs phenomenon in Fourier analysis, splines and wavelet approximations. Mathematics and its Applications, 446. Kluwer Academic Publishers, Dordrecht, 1998.

6. Gröchenig, K., Janssen, A., Kaiblinger, N., Norbert, P.: Note on B-splines, wavelet scaling functions, and Gabor frames. IEEE Trans. Inform. Theory. 49(12) (2003) 3318-3320

7. Liu, Y.: Irregular sampling for spline wavelet subspaces. IEEE Trans. Inform. Theory. 42 (1996) 623-627

8. Sun, W. C., Zhou, X. W.: Average sampling in spline subspaces. Appl. Math. Letter. 15 (2002) 233-237

9. Sun, W. C., Zhou, X. W.: Reconstruction of bandlimited signals from local averages. IEEE Trans. Inform. Theory. 48 (2002) 2955-2963

10. Unser, M., Blu, T.: Fractional splines and wavelets. SIAM Rev. 42(1) (2000) 43-67

11. Van De Ville, D., Blu, T., Unser, M., etc.: Hex-splines: a novel spline family for hexagonal lattices. IEEE Trans. Image Process. 13(6) (2004) 758-772

12. Walter, G. G.: Negative spline wavelets. J. Math. Anal. Appl. 177(1) (1993) 239253

13. Wang, J.: Spline wavelets in numerical resolution of partial differential equations, International Conference on Wavelet Analysis and its application, AMS/IP Studies in Advanced Mathematics. 25 (2002) 257-276 
14. Xian, J., Lin, W.: Sampling and reconstruction in time-warped spaces and their applications. Appl. Math. Comput. 157(1) (2004) 153-173

15. Xian, J., Luo, S. P., Lin, W.: Improved A-P iterative algorithm in spline subspaces. Lecture Notes in Computer Science. 3037 (2004) 60-67 